\title{
Assessment of load transfer characteristics of a fiber-reinforced titanium-matrix composite
}

\author{
U. Ramamurty * \\ Department of Metallurgy, Indian Institute of Science, C.V. Raman Avenue, Bangalore 560 012, India
}

\begin{abstract}
The extent of stress transfer that occurs around broken fibers dictates the longitudinal strength as well as the reliability of the fiber-reinforced composites. The governing load transfer characteristics of a $\sim 32-\mathrm{vol} \% \mathrm{SiC}$ fiber reinforced Ti-6Al-4V matrix composite (TMC) were investigated by recourse to uniaxial tension and 4-point bend flexure tests. Measured strengths and the variability in them are compared with the predictions of different load sharing models. The experimental tensile stress-strain response indicates to localization in fiber failure and the local load sharing (LLS) model predicts the composite strength more closely than a global load sharing (GLS) model. The flexure strengths are significantly larger than those predicted using the analytical models but are similar to that obtained using simulations that incorporate stress gradients and LLS. Broader distribution in flexure strength is also in agreement with simulation results, indicating that the LLS governs the strength of the TMCs. Implications of this observation are discussed. The TMCs are compared and contrasted with the Al-matrix composites in terms of their strength variability and size-scaling.
\end{abstract}

Keywords: A: Metal matrix composites; Fibers; B: Strength; C: Stress transfer; Statistics

\section{Introduction}

The longitudinal strength and reliability of continuous fiber-reinforced metal matrix composites (MMCs) are controlled by two factors: (i) the intrinsic strength characteristics of the fibers, dictated by the flaw distribution, and (ii) the extent of stress re-distribution that occurs around fiber breaks and its effect on subsequent fiber fracture [1]. The present article focuses on the latter problem. When both the fiber-matrix interface and the matrix itself are strong, the re-distribution of stress may occur very locally around the break location, over distances comparable to the fiber spacing. Conversely, if either the interface or the matrix are sufficiently weak, the re-distribution may occur equally over a longer

\footnotetext{
* Tel.: +91 802293 3241; fax: +91 8023600472

E-mail address: ramu@met.iisc.ernet.in.
}

transverse length scale, spanning many a fiber-spacing. This extreme situation is termed as global load sharing (GLS). The other extreme, wherein the stress of a broken fiber is shed only to its immediate neighbors, is one of strongly local load sharing (LLS). Intermediate degrees of load sharing can be expected in most MMCs of commercial interest.

The degree of load sharing plays a central role in the sequence of fiber breakage during tensile straining and hence on the composite strength and its variability. Notably, under GLS conditions, the composite strength is essentially deterministic, provided that the gauge length is greater than the characteristic load transfer length, $\delta_{\mathrm{c}}$, (defined in Section 2) and the number of fibers within the composite is sufficiently large $\left(\geqslant 10^{3}\right.$ to $10^{4}$ ) [2]. In contrast, under strongly LLS conditions, the fiber bundle strength is intrinsically stochastic and dependent upon the volume of material under stress 
[3]. In both cases, stochastic effects arise when the gauge length is smaller than $\delta_{\mathrm{c}}$ or when the number of fibers is small.

The LLS behavior is exemplified by the $\mathrm{Al}_{2} \mathrm{O}_{3}$-fiber reinforced $\mathrm{Al}$ alloys (without fiber coatings), described in [4]. In these systems, the matrix bonds strongly to the fibers. Moreover, for typical Al alloys (excepting high purity $\mathrm{Al}$ itself), the yield strength of the matrix is sufficiently high that the stress concentrations around fiber breaks cannot be completely eliminated by the attendant plastic straining in the vicinity of the break. The localized nature of the load sharing manifests in the volume-dependence of strength, as demonstrated by tests performed in uniaxial tension as well as 3- and 4-point flexure. Despite the LLS conditions operative in this system, the variability in the in situ fiber bundle strength is remarkably small, with a Weibull modulus of about 50 or, equivalently, a coefficient of variation of about $2 \%$.

The focus of the present paper is on a system comprising $\mathrm{SiC}$ fibers in a $\mathrm{Ti}$ alloy matrix (TMC). The $\mathrm{SiC}$ fibers are coated with $\mathrm{C}$ in order to inhibit chemical reactions between the fibers and the Ti matrix during consolidation. The $\mathrm{C}$ coatings are weak and promote interfacial debonding and sliding following fiber failure. This process is expected to alleviate the stress concentrations associated with the fiber breaks and hence the TMCs are considered, hitherto, as composites that obey GLS with a deterministic strength [5]. Preliminary experiments on a limited set of experiments, that too on a relatively small specimen volume range, reinforce this notion [6]. However, predictions made on composites that obey LLS suggest that for critical assessment of the load transfer characteristics, experiments on specimens with very large differences in volumes (at least two to three orders of magnitude difference) have to be conducted [7]. Furthermore, statistically significant number of experiments for any given specimen volume is necessary to obtain information about the strength variability, which in turn can be used to assess the governing load sharing mechanism.

The main objective of this work is to critically ascertain the load transfer characteristics between fiber and the matrix in the TMC system and establish whether the strength follows weakest link scaling laws, particularly in nonuniform loading conditions such as bending. This is accomplished by comparing and analyzing the strength and its variability in both uniaxial tension and 4-point flexure. The experimental measurements are analyzed in the context of existing models of fiber bundle failure subject to various load transfer characteristics, ranging from GLS to strongly LLS. The notable theoretical developments are attributable to Phoenix and Raj [8], Curtin [1,2,7,9] and their co-workers. The key features of the models are highlighted in the subsequent section. This is followed by a presentation of the experimental measurements on the $\mathrm{SiC} / \mathrm{Ti}$ composite and the corresponding analysis.

\section{Background}

Relevant theories of fiber fragmentation and bundle failure in unidirectionally reinforced MMCs are reviewed briefly, with emphasis on differences in behavior under LLS and GLS conditions. Detailed review of this topic is given by Curtin [1]. In both the cases, the extent of load transfer along the fiber length is governed mainly by the shear strength of the interface, $\tau$. In systems with weak interfaces, $\tau$ is controlled by the sliding resistance of the interface; in systems with well bonded interfaces, in contrast, it is controlled by the shear yield stress of the matrix, $\tau_{\mathrm{y}}$. This shear resistance also plays an important role in determining the extent of stress re-distribution to neighboring fibers: GLS conditions are favored when $\tau$ is sufficiently low. For both GLS and LLS conditions, the fiber bundle strength is dictated by a characteristic transfer length, $\delta_{\mathrm{c}}$, given by [2]

$\delta_{\mathrm{c}}=\frac{R S_{\mathrm{c}}}{\tau}=\left(\frac{\sigma_{0} R L_{0}^{1 / m}}{\tau}\right)^{m /(m+1)}$

and a characteristic strength, $S_{\mathrm{c}}$,

$S_{\mathrm{c}}=\left(\frac{\sigma_{0} \tau L_{0}}{R}\right)^{1 /(m+1)}$,

where $R$ is the fiber radius, $m$ is the Weibull modulus of the fibers, and $\sigma_{0}$ is the reference strength corresponding to a reference length, $L_{0}$.

GLS assumes that the load lost by a fiber due to its breakage and slippage is transferred equally to all the unbroken fibers in the cross-sectional plane of the break. For a composite whose length is much greater than $2 \delta_{\mathrm{c}}$ and contains very large number of fibers in the cross-section, Curtin [2] obtained analytical solutions for the in situ bundle strength, $\sigma_{\mathrm{b}}$, as

$\sigma_{\mathrm{b}}=S_{\mathrm{c}}\left(\frac{2}{m+2}\right)^{1 /(m+1)}\left(\frac{m+1}{m+2}\right)$.

Assuming an elastic, perfectly-plastic matrix, the postyield stress-strain response under isostrain condition, applicable for stressing of the unidirectional composite in the fiber direction, can be written as

$\sigma(\varepsilon)=f E_{\mathrm{f}} \varepsilon\left(1-\frac{1}{2}\left(\frac{E_{\mathrm{f}} \varepsilon}{S_{\mathrm{c}}}\right)^{m+1}\right)+(1-f) \sigma_{\mathrm{m}}^{\mathrm{y}}$,

where $f$ is the fiber volume fraction, $\sigma_{\mathrm{m}}^{\mathrm{y}}$ is the matrix yield stress, and $E_{\mathrm{f}}$ and $E_{\mathrm{m}}$ are the elastic modulus of the fiber and the matrix, respectively. From Eq. (4), the ultimate strength of the composite, $\sigma_{\mathrm{c}}^{\mathrm{u}}$ can be obtained as [1] 
$\sigma_{\mathrm{c}}^{\mathrm{u}}=f S_{\mathrm{c}}\left(\frac{2}{m+2}\right)^{1 /(m+1)}\left(\frac{m+1}{m+2}\right)+(1-f) \sigma_{\mathrm{y}}^{\mathrm{m}}$.

Note that the $\sigma_{\mathrm{c}}^{\mathrm{u}}$ thus obtained is a deterministic quantity and does not scale with the size of the composite.

Pheonix and Raj [8] considered the transverse scale of stress distribution to be much smaller than the cross-sectional dimension of the composite (i.e., no stress gradients within the vicinity of a broken fiber were considered, instead all the fibers within the bundle were assumed to carry the additional load shed on them due to the broken fiber equally) and estimated the composite strength and its variability using the chain of bundles concept with weak-link scaling originally proposed by Gücer and Gurland [10]. Close approximations of the mean strength, $\bar{\sigma}_{\mathrm{b}}$, and Weibull modulus, $m_{\mathrm{b}}$, of the in situ fiber bundle were obtained as [8]

$\bar{\sigma}_{\mathrm{b}}=\Gamma\left(1+\frac{1}{m_{\mathrm{b}}}\right) S_{\mathrm{c}}\left[\mu^{*}+\gamma^{*}\left(\frac{\ln (\ln N)+\ln (4 \pi)}{\sqrt{8 \ln N}}-\sqrt{2 \ln N}\right)\right]$

and

$m_{\mathrm{b}}=\frac{\mu^{*} \sqrt{2 \ln N}}{\gamma^{*}}+0.5[\ln (\ln N)+\ln (4 \pi)-4 \ln N]$,

where $\Gamma(\cdot)$ is the gamma function, $\mu^{*}$ and $\gamma^{*}$ are the mean strength and standard deviation of the finite-sized fiber bundle containing $n$ fibers and $N$ is the dimensionless composite size. For a given composite of volume $V, N$ can be obtained by using the relation [1]

$N=\frac{f V}{0.4 n \pi R^{2} \delta_{\mathrm{c}}}$.

Predicting the strength of a composite that obeys LLS by analytical means is a considerably more complicated problem, with the key task being the identification of the critical cluster of damaged fibers that leads to instability in the composite component [1]. This requires coupling of statistics with mechanics and close monitoring of the evolution of the fiber damage under the imposed LLS condition. Computer simulations of this problem by Zhou and Curtin [9], discretized and by employing the lattice Green's function method with tunable load sharing, led to an analytical model that connects failure under LLS and GLS and hence is capable of predicting the strength and its variability through weak link scaling. Notably, Ibnabdeljalil and Curtin [7] have shown that at some critical cluster or "link" size in LLS, the failure probability under LLS is identical to that obtained under GLS (i.e., Eqs. (6) and (7)) for the same size $n$. In other words, the strength and reliability of composite governed by LLS with a critical cluster size of $n_{l}$ (of length equal to $0.4 \delta_{\mathrm{c}}$ ) will be exactly the same as that of a composite governed by GLS with a finite-sized fiber bundle of size $n_{l}$. Once this identity is established, all that remains to be done is the identification of $n_{l}$ for a given LLS in a particular composite system and then determine the size-dependent composite strength and reliability using Eqs. (6)-(8). For very strong LLS, Ibnabdeljalil and Curtin [7] have found empirical correlations (on the basis of extensive numerical simulations) between $n_{l}$ and $m$ as

$n_{l}=403 m^{-1.28}$.

In the following, the results obtained on TMCs are analyzed within the framework of these models in order to assess the load sharing characteristics in it.

\section{Material and experiments}

The material used in this study was a Ti-6Al-4V alloy reinforced with $\sim 32 \mathrm{vol} \%$ of unidirectionally aligned Sigma $\mathrm{SiC}$ fibers (fiber radius, $R=50 \mu \mathrm{m}$ ). The panel consisted of 6 plies of fibers with a total thickness of $1.0 \mathrm{~mm}$. A micrograph of the cross-section through the composite is shown in Fig. 1. A particularly notable feature of the microstructure is the uniformity of the fiber distribution. This leads to the very narrow distribution in fiber volume fraction.

Uniaxial tensile tests were performed on straight bars with a 6-mm width and $75 \mathrm{~mm}$ gauge length. Stainless steel tabs were affixed to the specimen ends using epoxy. Tensile strains were measured using strain gauges that were bonded to the specimens. Four-point flexure tests were performed on $6 \mathrm{~mm}$ wide specimens. The loading direction was normal to the plane of the composite panel. The inner and outer loading spans were 9 and $27 \mathrm{~mm}$, respectively. Strains were measured on both the tensile and compressive faces using strain gauges. In total, 9 tension and 20 flexure tests were conducted.

The analysis of the strength measurements was based in part on previous measurements of other properties of the composite. These include the strength distribution of the fibers (extracted from the composite) [11]; the sliding resistance of the interface, measured by fiber pushout as well as pullout [11,12]; and the residual thermal stresses measured by a matrix

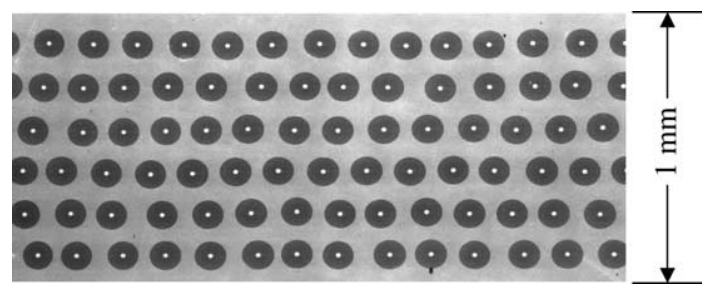

Fig. 1. A cross-sectional view of the SiC fiber reinforced TMC. 
Table 1

Relevant properties of fibers, matrix and composite

\begin{tabular}{|c|c|c|}
\hline Property & Value & Reference \\
\hline \multicolumn{3}{|l|}{ BP sigma fibers } \\
\hline Radius, $R$ & $50 \mu \mathrm{m}$ & \\
\hline Elastic modulus, $E_{\mathrm{f}}$ & $380 \mathrm{GPa}$ & {$[6]$} \\
\hline Reference strength, $\sigma_{0}$ & $1.47 \mathrm{GPa}$ & {$[6]$} \\
\hline Reference length, $L_{0}$ & $1 \mathrm{~m}$ & \\
\hline Weibull modulus, $m$ & 5.3 & {$[6]$} \\
\hline Mean strength of Fiber Bundle, $\mu^{*}$ & $0.749 S_{\mathrm{c}}$ & \\
\hline $\begin{array}{l}\text { Standard deviation in fiber } \\
\text { bundle strength, } \gamma^{*}\end{array}$ & $0.0297 S_{\mathrm{c}}$ & \\
\hline \multicolumn{3}{|l|}{ Ti-6Al-4V alloy matrix } \\
\hline Elastic modulus, $E_{\mathrm{f}}$ & $114 \mathrm{GPa}$ & {$[20]$} \\
\hline Yield stress, & $820 \mathrm{MPa}$ & \\
\hline Yield strain, & $0.72 \%$ & \\
\hline \multicolumn{3}{|l|}{ Composite } \\
\hline Number of fiber rows & 6 & \\
\hline Fiber volume fraction, $f$ & 0.326 & \\
\hline Elastic modulus, $E_{\mathrm{c}}$ & $200 \mathrm{GPa}$ & \\
\hline Residual strain in the fibers & $-0.152 \%$ & {$[13]$} \\
\hline \multicolumn{3}{|l|}{ Sliding stress, $\tau$} \\
\hline Push-in measurement & $130 \mathrm{MPa}$ & {$[5]$} \\
\hline Pull-out measurement & $60 \mathrm{MPa}$ & {$[11]$} \\
\hline \multicolumn{3}{|l|}{ Characteristic strength, $S_{\mathrm{c}}$} \\
\hline$\tau=130 \mathrm{Mpa}$ & $4817 \mathrm{MPa}$ & \\
\hline$\tau=60 \mathrm{MPa}$ & $4261 \mathrm{MPa}$ & \\
\hline \multicolumn{3}{|l|}{ Characteristic length, $\delta_{\mathrm{c}}$} \\
\hline$\tau=130 \mathrm{MPa}$ & $1.8528 \mathrm{~mm}$ & \\
\hline$\tau=60 \mathrm{MPa}$ & $3.5509 \mathrm{~mm}$ & \\
\hline
\end{tabular}

dissolution technique [13]. The key properties are summarized in Table 1.

The average fiber volume fraction, $\bar{f}$, was measured in a number of fractured test specimens. The measurements were made by counting the total number of fibers on the fracture surface, excluding the ones that had been cut at the edges during machining, and subsequently calculating the area fraction from the fiber diameter and the relevant cross-sectional area $(6 \mathrm{~mm} \times 1 \mathrm{~mm})$. Results of these measurements show that the fiber volume fraction distribution is extremely narrow, with the coefficient of variation of this distribution being $\sim 0.3 \%$. This result is consistent with the observed uniformity in the fiber distribution (Fig. 1). The average fiber volume fraction, $\bar{f}$ is 0.326 . Because of the minimal variation in $f$, it imparting a statistical distribution to the measured strength is assumed to be negligible and hence is ignored in the subsequent analysis.

Similarly, variability in the matrix strength affecting the composite strength statistics is ignored in view of the very low variability in the strength of ductile metals. Also, the strength of the matrix is assumed to be independent of the volume of the material being tested. As a result of these assumptions, the only parameter that affects the size scaling and variability of the composite strength will be the $\sigma_{\mathrm{b}}$.

\section{Results}

\subsection{Stress-strain response}

Typical tensile stress, $\sigma$, and the corresponding instantaneous tangent modulus, $\mathrm{d} \sigma / \mathrm{d} \varepsilon$, variation with tensile strain, $\varepsilon$, are shown in Figs. 2(a) and (b), respectively. The composite starts to deform elastically upon initial loading with an elastic modulus, $E_{\mathrm{c}}=198 \mathrm{GPa}$, a value consistent with that predicted using the ruleof-mixtures, $E_{\mathrm{c}}=f E_{\mathrm{f}}+(1-f) E_{\mathrm{m}}$. The onset of yielding occurs at $\sim 0.35 \%$ and the nonlinear behavior continues until a strain of $\sim 0.55 \%$. Upon further loading, the composite again deforms linearly but with a reduced slope that is $\approx f E_{\mathrm{f}}$, indicating that the fibers alone are carrying all the additional load at this stage of deformation. Progressive fiber failure, as reflected by a slight decrease in $\mathrm{d} \sigma / \mathrm{d} \varepsilon$, initiates at about $1400 \mathrm{MPa}$ and catastrophic fracture ensues almost immediately $(\sim 1482 \mathrm{MPa})$.

Typical nominal stress-displacement behavior under flexure is shown in Fig. 3(a). Measured tensile and
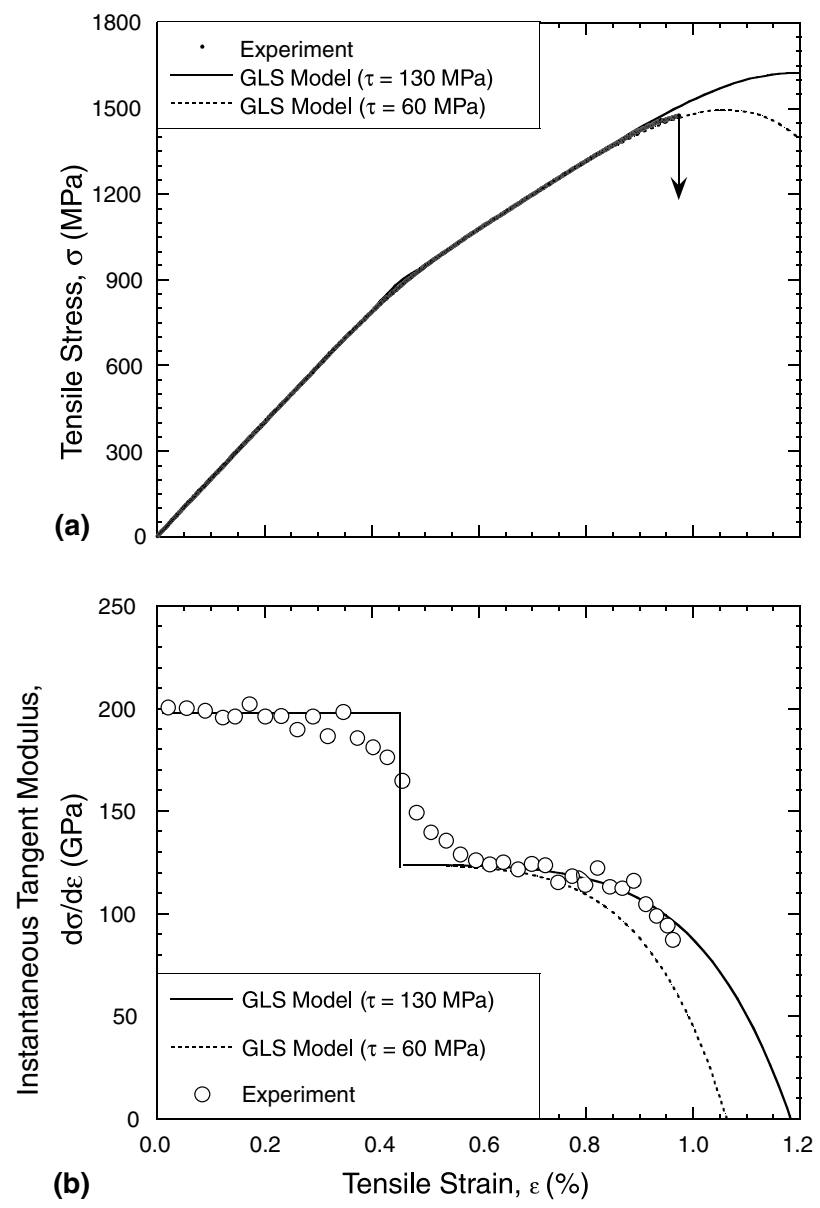

Fig. 2. (a) Typical tensile stress, $\sigma$, vs. strain, $\varepsilon$, response of the TMC. (b) Corresponding instantaneous tangent modulus, $\mathrm{d} \sigma / \mathrm{d} \varepsilon$, curve showing salient features of deformation. Predicted response using the GLS model is also plotted for comparison. 

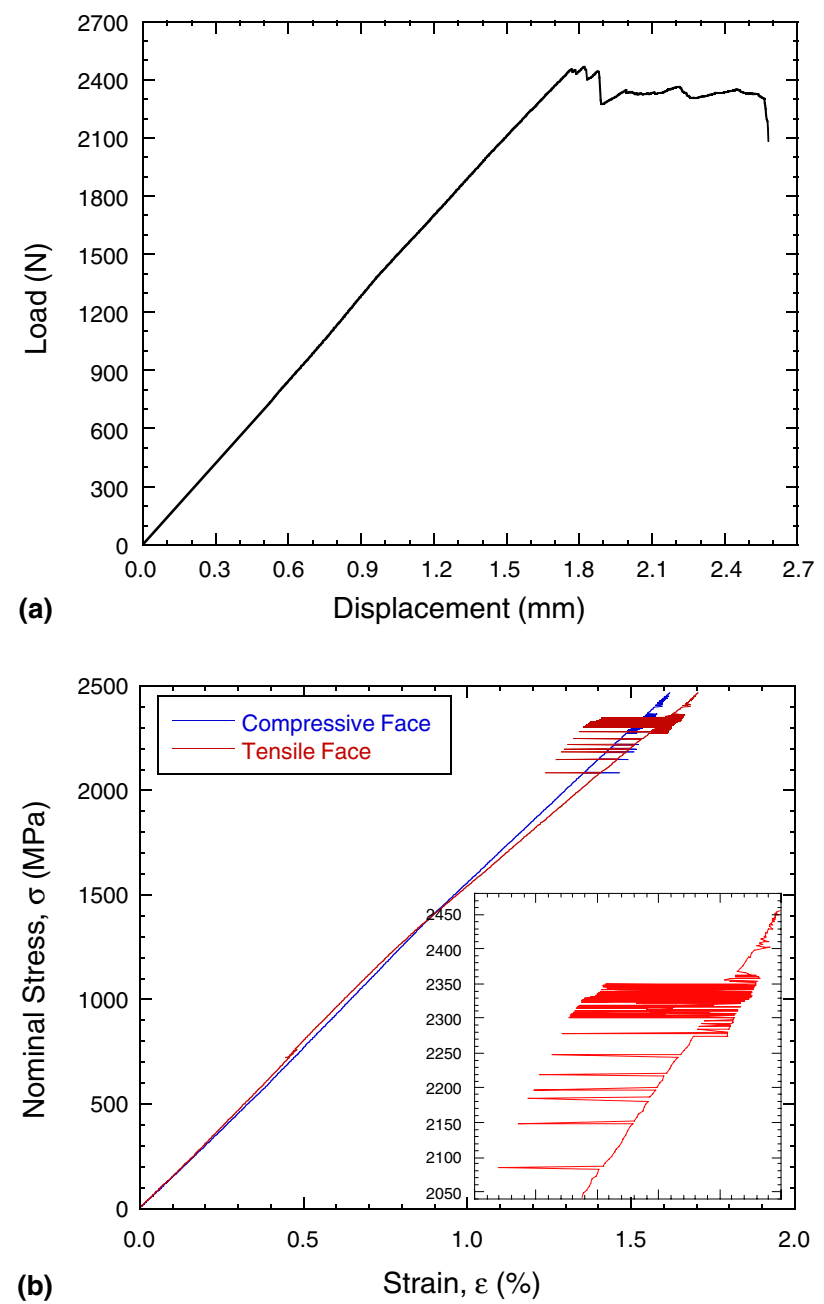

Fig. 3. (a) Typical load-displacement behavior of the TMC under flexure. (b) Tensile and compressive strains plotted against the nominal stress. Inset shows a magnified view of the fiber failure regime.

compressive strains are plotted against nominal stress (calculated by using Euler-Bernoulli beam theory for elastic materials) in Fig. 3(b). A couple of differences between the tensile and flexure responses are noteworthy. The load carrying capacity of the composite remains significant even after the first instability point. This discontinuous yet prolonged load carrying capacity beyond the first peak is due to initiation, propagation, and arrest of cracks caused by the bridging action of the fibers [14]. The first peak in the nominal stress, which also happens to be the maximum stress carried by the specimen, is taken as the strength of the composite.

The second observation pertains to the strength and failure strain (strain corresponding to the first peak) of the composite under flexure. They are considerably higher (by about $\sim 65 \%$ ) when compared to their respective values in tension. An extrinsic cause for this anomaly is the macroscopic stress redistribution due to the plastic flow of the matrix starting at the tensile face. Due to this nonlinear stress-strain response, the nomi- nal stress calculated on the basis of elastic beam theory overestimates the true stress acting along the tensile face. Another reason for the high strength in flexure could be the high stress gradients effecting the fiber fragmentation.

Note from Fig. 3(b) that at high stresses (close to the first peak stress) considerable noise in both the tensile and compression strain data is seen. Closer examination (inset of Fig. 3(b)) reveals that it is due to discrete strain jumps of about $0.16 \%$. (Aside, distinct "clicking" sounds at higher stresses could be heard while conducting these tests.) Clearly, these strain jumps are a result of the fiber fragmentation. The following are some of the observations.

(1) The first strain jump occurs at a nominal stress of $2084 \mathrm{MPa}$, followed by considerable fiber failure activity between 2300 and $2350 \mathrm{MPa}$.

(2) Above the stress level of $\sim 2370 \mathrm{MPa}$, the strain bursts completely stop and further stress build up without any softening is seen. This indicates that the fiber fragmentation in the first row has saturated and the second row of fibers starts experiencing the higher stresses. Furthermore, residual fiber pull out of the fragmented fibers aids in additional load carrying capability. This observation clearly implies that the saturation fragmentation of the first row of fibers in itself is insufficient to cause the specimen to fracture.

(3) Relatively muted strain bursts are seen again above the stress level of $2400 \mathrm{MPa}$, indicating that the second row of fibers have started fragmenting. Since the fragmenting fibers are farther away from the strain gauge on the tensile face, the amplitude of the strain bursts is relatively smaller.

\subsection{Strength variation}

Fig. 4 shows the variation in tensile and flexure strength of the composite, plotted against the cumulative probability of failure, $P$, calculated using the relation

$P=(l-0.5) / I$

where $l$ is the ascending rank of a particular data point in the data set of size $I$. The tensile strength distribution is very narrow, with a range of only $\sim 40 \mathrm{MPa}$ or $\sim 2.6 \%$. The mean strength of the composite, $\bar{\sigma}_{\mathrm{c}}$, is $1482 \mathrm{MPa}$ with a standard deviation of 14.2. The uncertainty in the standard deviation $(1 / \sqrt{2(9-1)}=0.25)$ is 3.6 $\mathrm{MPa}$. The mean tensile failure strain is $1.00 \%$ with a standard deviation of $0.0405 \%$.

The strength distribution in flexure is much broader (total range is $\sim 400 \mathrm{MPa}$ or $\sim 16 \%$ ) than that in tension. Here, $\bar{\sigma}_{\mathrm{c}}=2523 \mathrm{MPa}$ with a standard deviation of 


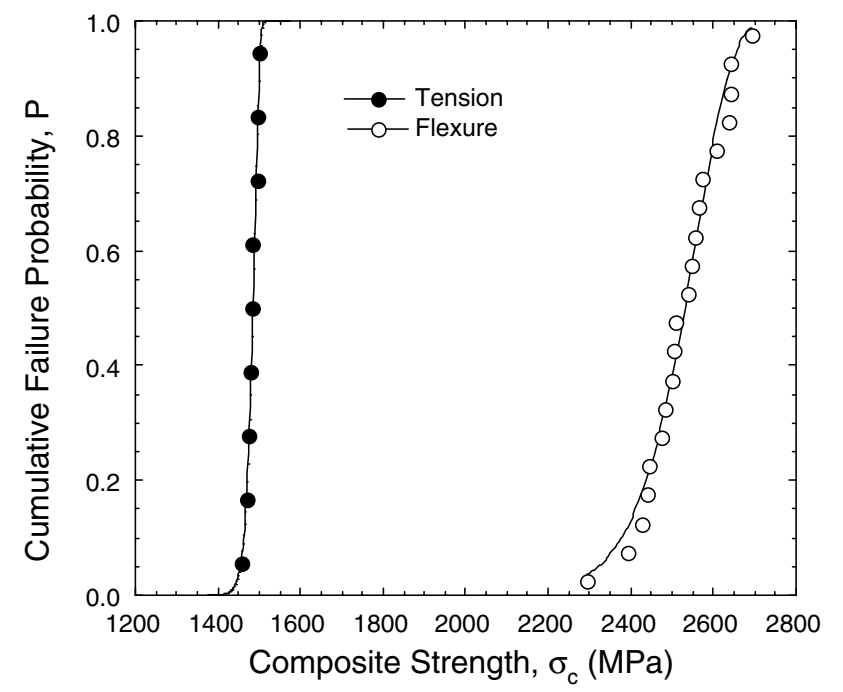

Fig. 4. Variation in tensile and flexure strengths of the composite plotted against the cumulative probability of failure, $P$.

$97.4 \pm 15.8 \mathrm{MPa}$. Higher strength and broader distribution of it vis-à-vis tensile test results confirm to the expected trends of higher strength and larger scatter in quasi-brittle specimens at smaller volumes. Average value of the strain to failure on the tensile face of the flexure specimen is $1.6921 \%$ with a standard deviation of $0.0646 \%$.

Distribution in $\sigma_{\mathrm{b}}$, obtained by subtracting the matrix contribution from $\sigma_{\mathrm{c}}^{\mathrm{u}}$, are fitted with Weibull distribution function,
$P(\sigma)=1-\exp \left[-\frac{V}{V_{0}}\left(\frac{\sigma}{\sigma_{0}}\right)^{m_{\mathrm{c}}}\right]$,

where $V$ is the specimen volume, $V_{0}$ is the reference volume, $\sigma_{0}$ is the reference strength and $m_{\mathrm{b}}$ is the Weibull modulus of the fiber bundle. Extracted values of $m_{\mathrm{b}}$ are listed in Tables 2 and 3.

\section{Discussion}

\subsection{Uniaxial tension}

The axial tensile stress-strain response, $\sigma_{\mathrm{c}}(\varepsilon)$, of a unidirectional TMC can be modeled using the rule-of-mixtures $[5,15]$

$\sigma_{\mathrm{c}}(\varepsilon)=f \sigma_{\mathrm{f}}(\varepsilon)+(1-f) \sigma_{\mathrm{m}}(\varepsilon)$,

where $\sigma_{\mathrm{f}}(\varepsilon)$ and $\sigma_{\mathrm{m}}(\varepsilon)$ represent the average axial stresses in the fiber and the matrix, respectively. The matrix is assumed to be elastic-perfectly plastic, reasonable for Ti-alloys. Hence,

$$
\begin{aligned}
\sigma_{\mathrm{m}}(\varepsilon) & =E_{\mathrm{m}} \varepsilon \quad \varepsilon \leqslant \varepsilon_{\mathrm{c}}^{\mathrm{y}}, \\
& =E_{\mathrm{m}} \varepsilon_{\mathrm{c}}^{y} \quad \varepsilon \geqslant \varepsilon_{\mathrm{c}}^{\mathrm{y}}
\end{aligned}
$$

with $\varepsilon_{\mathrm{c}}^{\mathrm{y}}$ being the composite yield strain. Note that in the absence of any residual strains, $\varepsilon_{\mathrm{c}}^{\mathrm{y}} \equiv \varepsilon_{\mathrm{m}}^{\mathrm{y}}$, the unconstrained matrix yield strain. However, residual strains arise upon cooling the composite from the processing temperature because of the large thermal expansion mis-

Table 2

\begin{tabular}{|c|c|c|c|c|c|c|}
\hline \multirow[b]{2}{*}{$\tau(\mathrm{MPa}) \rightarrow$} & \multicolumn{2}{|c|}{ Number of Links } & \multicolumn{2}{|c|}{$\begin{array}{l}\text { Mean composite strength } \\
(\mathrm{MPa})\end{array}$} & \multicolumn{2}{|c|}{$\begin{array}{l}\text { Fiber bundle Weibull } \\
\text { modulus }\end{array}$} \\
\hline & 130 & 60 & 130 & 60 & 130 & 60 \\
\hline $\operatorname{GLS}(n=\infty)$ & - & - & 1656 & 1529 & $\infty$ & $\infty$ \\
\hline GLS (finite $n$ ) & 101 & 53 & 1609 & 1496 & 69 & 65 \\
\hline LLS & 525 & 273 & 1585 & 1474 & 79 & 75 \\
\hline LLS (periodic BC) & - & - & 1572 & 1494 & 129 & 107 \\
\hline Experiment & \multicolumn{2}{|c|}{-} & \multicolumn{2}{|c|}{$1482 \pm 14$} & \multicolumn{2}{|c|}{$\begin{array}{c}68 \pm 10 \\
23 \pm 4 \text { (failure strain) }\end{array}$} \\
\hline
\end{tabular}

Experimental and predicted tensile strength and its Weibull modulus of the TMC

Table 3

\begin{tabular}{|c|c|c|c|c|c|c|}
\hline \multirow[b]{2}{*}{$\tau(\mathrm{MPa}) \rightarrow$} & \multicolumn{2}{|c|}{ Number of links } & \multicolumn{2}{|c|}{$\begin{array}{l}\text { Mean in situ fiber bundle } \\
\text { strength (MPa) }\end{array}$} & \multicolumn{2}{|c|}{$\begin{array}{l}\text { Fiber bundle Weibull } \\
\text { modulus }\end{array}$} \\
\hline & 130 & 60 & 130 & 60 & 130 & 60 \\
\hline $\operatorname{GLS}(n=\infty)$ & - & - & 3385 & 2994 & $\infty$ & $\infty$ \\
\hline GLS (finite $n$ ) & 12 & 6 & 3301 & 3018 & 52 & 46 \\
\hline LLS & 10 & 5 & 3362 & 3031 & 51 & 43 \\
\hline LLS (simulation) & - & - & 5070 & 4484 & 35 & 32 \\
\hline Experiment & \multicolumn{2}{|c|}{-} & \multicolumn{2}{|c|}{4163} & \multicolumn{2}{|c|}{$\begin{array}{c}16 \pm 1 \\
54 \pm 9 \text { (failure strain) }\end{array}$} \\
\hline
\end{tabular}

Experimental and predicted in situ bundle strengths and its Weibull modulus for the 4-point bend tests 
match between the fibers and the matrix [13]. In the asprocessed state, the matrix is under tension whereas the fibers are in compression. Because of the preexisting axial tensile residual strains, $\varepsilon_{\mathrm{m}}^{R}$, in the matrix, its tensile yield strain decreases by that amount. Thus,

$\varepsilon_{\mathrm{c}}^{\mathrm{y}}=\varepsilon_{\mathrm{m}}^{\mathrm{y}}-\varepsilon_{\mathrm{m}}^{R}$.

Residual strain in the fibers, measured in this TMC using the selective etching technique is $\sim-0.15 \%$ [13]. Corresponding residual strains in the matrix (calculated) are $\sim 0.27 \%$. From Fig. 2(b), it is seen that $\varepsilon_{\mathrm{c}}^{\mathrm{y}} \sim 0.45 \%$. Therefore, $\varepsilon_{\mathrm{m}}^{\mathrm{y}} \sim 0.72$ which is similar to the typical values for the yield strain of Ti-4Al-6V alloy of $\sim 0.7 \%$. Note also that the matrix yield strength, $\sigma_{\mathrm{m}}^{\mathrm{y}}$ (estimated by using an $E_{\mathrm{m}}$ of $\left.114 \mathrm{GPa}\right) \sim 820 \mathrm{MPa}$, is also consistent with those typically reported for Ti-6Al-4V alloys [15].

The predicted post-yield stress-strain response of the composite, using Eqs. (3) and (12), is plotted in Fig. 2. Here, the fiber failure statistics measured by Weber et al. [5] on fibers extracted from this TMC by dissolving the matrix away and the fiber sliding resistance measured using fiber push-out tests ( $\tau=130 \mathrm{MPa})$ are used. However, push-out tests tend to overestimate the value $\tau$ because of the Poisson expansion of the fibers during the test. Pull-out tests do not suffer from this drawback and hence give a better estimate of the value for $\tau$ since they represent the stress state in the fiber during a tensile test. Pull-out tests on a slightly different TMC by Walls and Zok [11] yield a $\tau$ of $\sim 60 \mathrm{MPa}$. Predictions made using this $\tau$ value are also tabulated.

As seen from Fig. 2(b), the model predictions are commensurate with the experimental trends. However, a couple of differences are noteworthy.

- Although the predicted and experimental strengths appear to be in good agreement, the failure strains are not in such a good agreement ( $\sim 10 \%$ difference). One of the reasons for the difference in failure strains could be a faster localization (or clustering) of fiber breaks. From Fig. 2(b), a small drop in $\mathrm{d} \sigma / \mathrm{d} \varepsilon$ just before fracture is noticeable. Thus far the experimental trends are commensurate with the GLS model predictions; indicating a small amount of non-interactive fiber failure. However, catastrophic fracture ensues much before the experimental $\mathrm{d} \sigma / \mathrm{d} \varepsilon$ reduces to 0 , indicating that localization initiates readily in this composite.

- The model predicts a sharp drop in $\mathrm{d} \sigma / \mathrm{d} \varepsilon$ at the composite yield point whereas the experimental results show a gradual transition. This discrepancy arises because the model assumes the matrix to be elasticperfectly plastic and that the residual stresses are distributed uniformly. However, in reality, the Ti alloys exhibit a slight hardening behavior [16]. Variation in the residual stresses as a function of distance away from the fiber could also lead to a gradual yielding behavior [17]. Since our primary interest is in the near fracture regime, which occurs at a much higher stress level beyond yielding of the composite, we ignore the gradual transition at yield.

Turning attention to the tensile strength and its variability, experimentally measured mean composite strength and fiber bundle Weibull modulus are compared with those predicted using different models in Table 2. For predicting the strength using the GLS model of Phoneix and Raj [8], a priori knowledge of the characteristic fiber bundle size, $n$, is necessary. Here, we consider $n$ to be equal to the number of fibers in the tensile specimen cross-section $(=240)$. Note that, this approach reduces the Phoneix and Raj model, in effect, to a GLS model corrected for finite bundle sizes. All the predictions are made using two values of $\tau, 130$ and $60 \mathrm{MPa}$, obtained using push-out and pull-out techniques.

It is seen from Table 2 that all the predicted mean strengths using $\tau=130 \mathrm{MPa}$ are higher than the experimentally measured value. For the case of $\tau=60 \mathrm{MPa}$, Phoneix and Raj model as well as the LLS model predict a mean strength that is very close to the experimentally measured strengths. All the predicted $m_{\mathrm{b}}$ are very high, 65 and above, suggesting a narrow distribution in the strength, consistent with the experimental observation. In particular, both the LLS model and the GLS model with finite specimen size correction predict an $m_{\mathrm{b}}$ that is close to the experimentally measured value of 68 . It is interesting to note that the Weibull modulus for the composite failure strain in tension is much smaller, $\sim 23 \pm 4$. Because of the gradual "flattening" of the stress-strain curve predicted by the GLS model at its maximum (see Fig. 2(a)), the distribution in failure stresses will be narrower than that in failure strains. Clearly, a larger distribution in the latter implies to localization (as exemplified by the experimental $\mathrm{d} \sigma / \mathrm{d} \varepsilon$ data in Fig. 2(b)) and hence it is reasonable to conclude that LLS model is more appropriate for describing the strength statistics of the TMC.

\subsection{Flexure response}

The flexure strengths obtained are analyzed to extract $\sigma_{\mathrm{b}}$, with the following two approaches.

1. Stress partitioning: Linearly partition the applied bending moment, $M$, into two components, $M_{\mathrm{f}}$ and $M_{\mathrm{m}}$, bending moments pertaining to the fibers and the matrix respectively. Assuming that the elastic-perfectly plastic matrix is fully yielded (reasonable, considering the fact that the failure strain of the flexure specimens is $\sim 2.5$ times the matrix yield strain), $M_{\mathrm{m}}=1.5 M_{\mathrm{E}}$, where $M_{\mathrm{E}}$ is the maximum elastic moment that the matrix can support and taking the 
yield stress of the matrix as $820 \mathrm{MPa}$, the mean in situ fiber bundle strength (extracted from the average flexure strength of $2520 \mathrm{MPa}$ ) is obtained as $5184 \mathrm{MPa}$.

2. Strain partitioning: Alternatively, assume a linear strain distribution through the composite thickness and hence calculate the strain in the first row of the fibers to be equal to 0.78 times the strain on the tensile face, yielding an in situ bundle strength of $4980 \mathrm{MPa}$.

For predicting $\sigma_{\mathrm{b}}$ using the various models presented in Section 2, we recognize that in essence the 4-point bend tests are tantamount to tensile testing of a composite of length $9 \mathrm{~mm}$ (inner span of the flexure specimen) and $6 \mathrm{~mm}$ (width of the specimen) in tension with single row of fibers in it. Predicted $\sigma_{\mathrm{b}}$ and its variability (in terms of the Weibull modulus) are listed in Table 3. As seen, both the values of the bundle strengths extracted from the experimental flexure results through the simple analyses are very large when compared to those predicted. This is clearly a consequence of the inability to analytically model the stress distribution due to matrix yielding as well as fiber breakage in flexure loading. Exact analysis of the flexure test results and comparison of the experimental and predicted stressstrain behavior is complicated by the following factors.

1. Relatively large size of the fibers vis-à-vis the specimen thickness, which leads to a large strain gradient across the fibers. Also, the small number of fibers across the specimen thickness (6) renders the application of continuum elasto-plastic stress analysis of the flexure beams difficult.

2. High strength of the matrix and hence significant contribution of it to the overall strength of the composite. Incorporating plasticity of the matrix at the same time as stochastic fiber bundle response to extract the stress state at failure analytically is difficult.

Foster [18] has performed numerical simulations on identical geometries to the tensile and 4-point bend flexure tests under LLS conditions, incorporating periodic boundary conditions (mimicking the tensile samples with six fibers in the cross-section) as well as stress gradients (mimicking the flexure specimens). The failure criterion for bending was considered to be formation of a half-plane of broken fibers below the center of the composite. Foster's predictions, based on 50 simulations of tensile geometry and 35 simulations of flexure geometry, are also given in Tables 2 and 3, respectively. It is seen that from Table 2 that the results of the simulations under LLS and with periodic boundary conditions are similar to those predicted using the analytical LLS model. Foster observed, on the basis of hundreds of simulations performed on composites with $4 \times 26$ lattice of fibers and length equal to $2 \delta_{\mathrm{c}}$ with periodic, semi-free and free boundaries, that the mean fiber bundle strengths are well within a standard deviation of one another and hence concludes that for a given $m$, the effect of boundary conditions on the fiber bundle strength is statistically insignificant. This is possibly the reason for the observed similarity in the simulation and analytical predictions.

Turning attention to the flexure tests, it is seen from Table 3 that the analytical and simulated LLS results

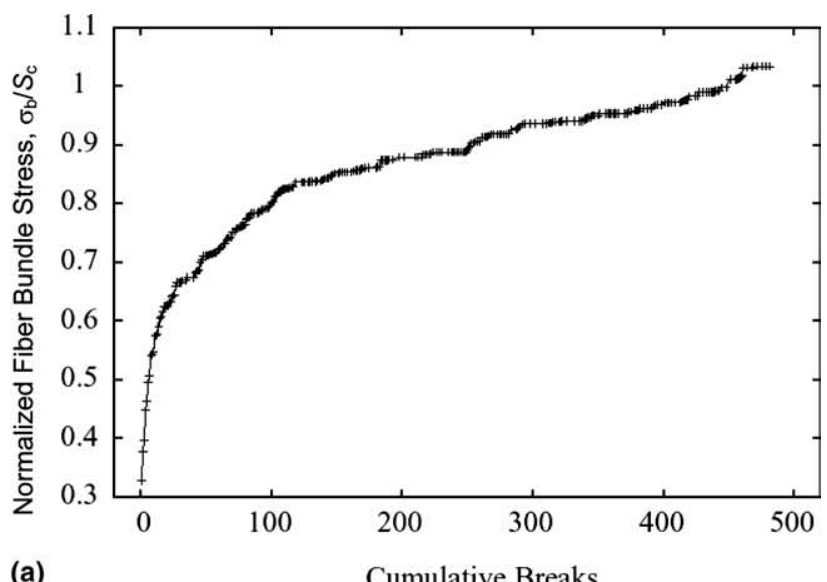

(a)

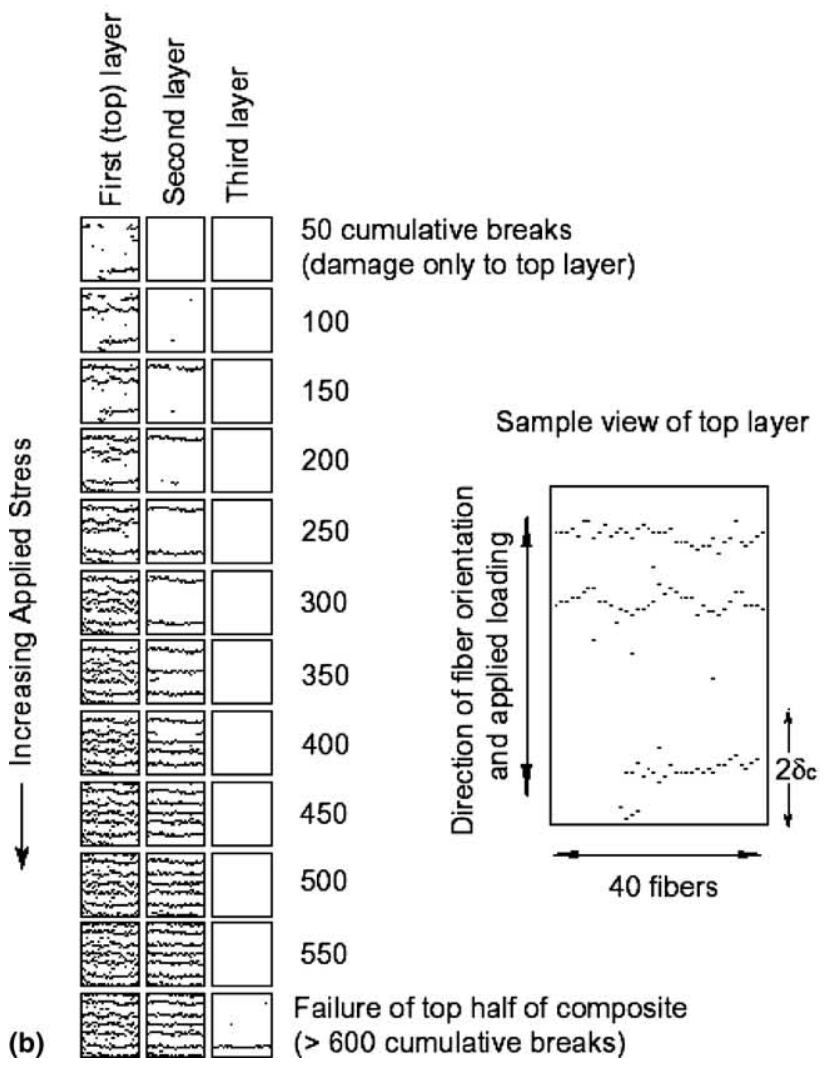

Fig. 5. (a) Plot of cumulative fiber failures vs. applied fiber bundle stress (normalized with the characteristic bundle stress, $S_{\mathrm{c}}$ ) under 4point bending in a LLS simulation. (b) Corresponding evolution of composite failure showing fiber breaks in top three layers of the composite that is subjected to increasing 4-point bending stress. 
(the latter incorporating the stress gradients associated with the flexure) diverge significantly. Furthermore, the simulation results are in very good agreement with the experimental results, especially the predicted $m_{\mathrm{c}}$. Note that the LLS model with a $\tau=60 \mathrm{MPa}$ also appears to predict a $m_{\mathrm{c}}$ that is reasonably close to the experimentally observed $m_{\mathrm{c}}$, the latter value unaffected by the complications in the load redistribution.

A key observation made during the LLS simulation of the flexure test by Foster [18] is that the composite is capable of sustaining much more damage even after the breakage of first layer of fibers. Fig. 5(a) shows the cumulative fiber failures plotted against the fiber bundle stress (normalized with the characteristic stress, $S_{\mathrm{c}}$ ) in a typical simulation of the flexure test. Each datum on this plot represents a fiber break. Fig. 5(b) shows the corresponding evolution of the damage in the top three layers of the flexure specimens. (Recall that the specimen has 6 fiber rows and hence Fig. 5(b) represents the fiber rows on the tensile side of the specimen.) As seen from Fig. 5(a), failure of the first row of fibers in the composite specimen occurs at $\sim 0.89 S_{\mathrm{c}}$ with approximately 200 fiber breaks. In general these simulations are consistent with the experimental observations of Fig. 2(b). While this stress itself is much higher than those predicted using the GLS and LLS models $\left(\sim 0.70 S_{\mathrm{c}}\right)$, further "hardening" of the composite is seen and it appears that the stress-fiber break plot flattens out asymptotically until the fiber breaks have reached the midsection of the specimen (Fig. 5(b)). While the exact failure criterion beyond this point is difficult to implement, it is clear from Fig. 5(a) that the high stress gradients present in the flexure specimens leads to considerably higher strength, broadly corroborating with the experimental results. These simulation results also imply that the LLS model is far better equipped, albeit requiring simulations of the exact geometry at times, to capture the actual material behavior.

\section{3. $T M C$ vs. $A M C$}

The Ti-matrix composite examined in this work is distinctly different from the $\sim 70 \mathrm{vol} \% \mathrm{Al}_{2} \mathrm{O}_{3}$ fiber reinforced Al-matrix composite (AMC) that we have examined in an earlier paper. In particular, the TMC differs from the AMC in three important respects as following.

- First, the yield strength of the Ti-alloy represents a significant fraction $(\sim 0.5)$ of the ultimate tensile strength (UTS) of the composite. Consequently, the tensile response of the composite is bilinear, with the change in slope occurring at roughly one half of the failure strain. An additional consequence is the development of non-linearity in the stress distribution across a flexure specimen following matrix yielding. Such effects must be taken into consideration in evaluating the true tensile stress within the fibers at fail- ure. In contrast, in the AMC, the yield strength of the matrix alloy is negligible ( $<5 \%$ of the UTS). Consequently, the tensile response is essentially linear up to fracture, and, in flexure, the stress distribution remains linear [4].

- Second, the diameter of the SiC fibers is about an order of magnitude larger than that of the $\mathrm{Al}_{2} \mathrm{O}_{3}$ fibers. The fiber size has two implications. (i) Under flexural loading of the TMC, substantial strain gradients occur over distances comparable to the fiber spacing. Moreover, the fibers closest to the tensile face experience a strain that is considerably lower than that in the matrix on the tensile face. In the AMC, the gradients that occur about a fiber spacing are negligibly small. (ii) The number of fibers found in a typical TMC tensile specimen is only $\sim 200$, compared to $\sim 20,000$ for a similarly-sized AMC specimen (the number ratio scaling with the inverse square of the ratio of fiber diameters). In the TMC flexural specimens, the effective number of fibers controlling strength is lower yet $(<100)$. Such small numbers can cause additional stochastic effects in the fiber bundle strength.

- Third, fiber coatings similar to that of $\mathrm{C}$ coating of $\mathrm{SiC}$ fiber in the TMC have not been utilized extensively in the AMC because of their role in reducing the transverse strength to extremely low levels. In the absence of coatings, the AMC interface is strong, leading to strongly LLS characteristics.

Despite these significant differences, TMCs and AMCs both obey the LLS model predictions with the size-scaling of their strength and reliability conforming to the analytical models developed by Ibnabdeljalil and Curtin [7]. This is perhaps due to the relatively high value of $\tau$ in both the cases, given by the interfacial sliding resistance for the TMC and by the matrix shear yield stress for the AMC. Another factor could be the large size of the fibers vis-à-vis the specimen size, leading a stress concentration analogous to that present at a notch as per fracture mechanics, leading to failure localization. However, the spatial as well as the specimen-to-specimen variations is fiber volume fraction are the additional considerations that have to be taken into consideration for understanding the AMC strength variability $[4,19]$.

\subsection{Implications}

Experimental results presented in this paper clearly demonstrate that there is an intrinsic size-scaling to the strength of the fiber reinforced MMCs. This size-scaling that arises due to the LLS conditions that prevail in these composites is particularly important when a large MMC component's strength has to be predicted on the basis of strength data generated using small coupons such as 
those used in our flexure tests on TMCs. Comparisons of the experimental data with predictions made using available models clearly show that the GLS models, while successful on occasion, can lead to erroneous predictions. For example, the GLS model predicts a mean strength of $1529 \mathrm{MPa}$ for tensile samples used in this study and hence appears to be in reasonable accordance with the experimentally measured value of $1482 \mathrm{MPa}$ (only $\sim 3 \%$ difference). The observed variability in tensile strength also appears to show that it is a deterministic quantity. However, for a component containing 100,000 links (a connecting rod with a $25-\mathrm{mm}$ diameter and $20 \mathrm{~cm}$ length), the TMC will have $1416 \mathrm{MPa}$ strength (as per LLC model), and hence the GLS would be over-predicting the strength by $\sim 10 \%$. For very small components sizes, GLS not only underestimates the strength but also predicts a higher reliability, which can lead to non-conservative designs. The GLS model also suffers from the fact that it overestimates that failure strains, which have important implications in fracture related properties of the composite such as its notch sensitivity.

The Phoneix and Raj model, while appears to be as good as the LLS model in terms of predicting both the tensile and flexure strengths as well as their variability, suffers from the implicit drawback that it does not offer any means to estimate the characteristic bundle size. The approach we have used in this work to employ this model, namely taking $n$ to be equal to the number of fibers in the specimen cross-section, will not work when the specimen size is large. As $n \rightarrow \infty$ the predictions will be identical to that made using the GLS model.

In view of the above constraints suffered by the GLS model and its variants, the LLS model appears to best model to describe the mechanical behavior of the TMC investigated in this work as well as the AMCs examined elsewhere [4]. Furthermore, good agreement between the experimental results and LLS model predictions imply that the load sharing between the fragmented fibers and its neighbors tends to be local in nature. However, it should be noted that these analytical models could only capture the strength of the composites when there is homogeneous loading (i.e., where there is uniform stress such as the uniaxial tension) with a reasonable degree of accuracy. Among the models available, the analytical LLS model appears to give most accurate results. However, it appears that for complex geometries and large stress gradients (such as flexure loading), computational simulation is the only recourse that is available. This is particularly true when the complexity gets compounded by the discreteness in the material system (like the presence of only a few fiber rows across the specimen thickness). This is precisely the reason for the lack of complete failure in Foster's simulations, only a stress region over which damage accumulates very rapidly, after which it slows down due to the constraints of equilibrium in the discrete sys- tem. What exactly is the failure criterion that has to be employed in such scenarios for predicting the fracture of the structure thus remains an open question.

In closing, it should be noted that the LLS model employed in this paper for the analysis of the experimental results is that developed Curtin and coworkers who treat the statistics of failure in terms of a chain of Gaussian bundles. Phoenix and Beyerlein [21] treat this problem differently, which leads to a fundamentally different form of distribution altogether, at least for moderate to larger fiber Weibull modulus values. However, while mathematically different, the predictions of these two LLS models are very similar.

\section{Conclusions}

The strength and its variability of a $\sim 32-\mathrm{vol} \% \mathrm{SiC}$ fiber reinforced Ti-6Al-4 V matrix composite, measured by conducting both uniaxial tension as well as 4-point bend flexure tests, suggest that the final failure of this composite is governed by the local load sharing. The experimental tensile stress-strain response indicates to localization in fiber failure as expected under LLS conditions. The flexure strengths are significantly larger than those predicted using the analytical models but are similar to that obtained using simulations that incorporate stress gradients and LLS. Similarly, distribution of the flexure strengths is broader, in agreement with simulation results. This work also highlights the possible problems involved in deciphering the data generated by flexure testing of composites and in turn connecting them with those tests conducted in a much simpler stress states.

\section{Acknowledgement}

It is indeed a pleasure to acknowledge Frank Zok of UCSB and Bill Curtin of Brown University for many useful discussions and insights. Thanks are also due to Glenn Foster, formerly at VPISU and now at UIUC, for his permission to use Figs. 5(a) and (b) (of this paper) from his MS thesis.

\section{References}

[1] Curtin WA. Adv Appl Mech 1999;36:163.

[2] Curtin WA. J Am Ceram Soc 1991;74:2837.

[3] Zweben C, Rosen BW. J Mech Phys Solids 1970;18:189.

[4] Ramamurty U, Zok FW, Leckie FA, Deve H. Acta Mater 1997;45:4603.

[5] Jansson S, Deve H, Evans AG. Metall Trans A 1991;22:2975.

[6] Weber CH, Chen X, Connell SJ, Zok FW. Acta Metall Mater 1994;42:3443.

[7] Ibnabdeljalil M, Curtin WA. Inter J Solids Struct 1997;34:2649.

[8] Phoenix SL, Raj R. Acta Metall Mater 1992;40:2813. 
[9] Zhou SJ, Curtin WA. Acta Metall Mater 1995;43:3093.

[10] Gücer DE, Gurland J. J Mech Phys Solids 1962;10:365.

[11] Walls DP, Zok FW. Acta Metall Mater 1994;42:2675.

[12] Connell SJ, Zok FW. Acta Mater 1997;45:5203.

[13] Ramamurty U, Dary FC, Zok FW. Acta Mater 1996;44:3397.

[14] Massabò R, Cox BN. J Mech Phys Solids 1999;47:1265.

[15] Ramamurty U, Zok FW, Leckie FA. Mater Sci Eng A 1996;214:62.

[16] Maykuth DJ, Monroe RE, Favor RJ, Moon DP. Titanium-base alloys: 6Al-4V. Columbus (OH): Batelle Memorial Institute; 1971.

[17] Nakamura T, Suresh S. Acta Metall Mater 1993;41:1665.
[18] Foster G. Tensile and Flexure Strength of Unidirectional FiberReinforced Composites: Direct Numerical Simulations and Analytic Models. Thesis for Masters Science in Engineering Mechanics, Virginia Polytechnic Institute and State University, Blacksburg, VA, USA; 1998.

[19] Ramamurty U, Seshacharyulu T. Mater Sci Eng A 1999;268:97.

[20] Nimmer RP, Bankert RJ. GE CRD Report No. 91, CRD 060; 1992.

[21] Phoenix SL, Beyerlein IJ. Statistical strength theory for fibrous composite materials. In: Kelly A, Zweben C, editors-in-chief, Chou TW, editor. Comprehensive composite materials (6 volumes), vol. 1, Pergamon, Elsevier Science; 2000 [chapter 1.19]. 